\title{
Deep learning neural networks for the third-order nonlinear Schrödinger equation: Solitons, breathers, and rogue waves
}

\author{
Zijian Zhou and Zhenya Yan* \\ Key Laboratory of Mathematics Mechanization, Academy of Mathematics and Systems Science, \\ Chinese Academy of Sciences, Beijing 100190, China \\ School of Mathematical Sciences, University of Chinese Academy of Sciences, Beijing 100049, China
}

\begin{abstract}
The third-order nonlinear Schrödinger equation (alias the Hirota equation) is investigated via deep leaning neural networks, which describes the strongly dispersive ion-acoustic wave in plasma and the wave propagation of ultrashort light pulses in optical fibers, as well as broader-banded waves on deep water. In this paper, we use the physics-informed neural networks (PINNs) deep learning method to explore the datadriven solutions (e.g., soliton, breather, and rogue waves) of the Hirota equation when the two types of the unperturbated and unperturbated (a $2 \%$ noise) training data are considered. Moreover, we use the PINNs deep learning to study the data-driven discovery of parameters appearing in the Hirota equation with the aid of solitons.
\end{abstract}

Keywords: Third-order nonlinear Schrödinger equation; deep learning; neural network; data-driven solutions

\section{Introduction}

As a fundamental and prototypical physical model, the one-dimensional cubic nonlinear Schrödinger (NLS) equation in the dimensionless form is

$$
i q_{t}+q_{x x}+2|q|^{2} q=0, \quad(x, t) \in \mathbb{R}^{2},
$$

where $q=q(x, t)$ denotes the complex field, and the subscripts stand for the partial derivatives with resect to the variables. Eq. (1) can be used to describe the wave propagation in many fields of Kerr nonlinear and dispersion media such as plasmas physics, deep ocean, nonlinear optics, Bose-Einstein condensate, and even finance (see, e.g., Refs. [1-18] and references therein). When the ultra-short laser pulse (e.g., 100 fs [17]) propagation were considered, the study of the higher-order dispersive and nonlinear effects is of important significance, such as third-order dispersion, self-frequency shift, and self-steepening arising from the stimulated Raman scattering [19-21]. The third-order NLS equation (alias the Hirota equation [22]) is also fundamental physical model. The Hirota equation and its extensions can also be used to describe the strongly dispersive ion-acoustic wave in plasma [23] and the broader-banded waves on deep ocean [24,25]. The Hirota equation is completely integrable, and can be solved via the bi-linear method [22], inverse scattering transform [26,27], and Darboux transform (see, e.g., Refs. [28-33]), and etc. Recently, we numerically studied the spectral signatures of the spatial Lax pair with distinct potentials (e.g., solitons, breathers, and rogue waves ) of the Hirota equation [34].

Up to now, artificial intelligence (AI) and machine learning (ML) have been widely used to powerfully deal with the big data, and play an more and more important role in the various fields, such as language translation, computer vision, speech recognition, and so on $[35,36]$. More recently, the deep neural networks were presented to study the data-driven solutions and parameter discovery of nonlinear physical models [37-52]. Particularly, the physics-informed neural networks (PINNs) technique $[41,46,52]$ were developed to study nonlinear partial differential equations. In this paper, we would like to extend the PINNs deep learning method to investigate

\footnotetext{
*Corresponding author. Email address: zyyan@mmrc.iss.ac.cn
} 
the data-driven solutions and parameter discovery for the focusing third-order nonlinear Schrödinger equation (alias the Hirota equation) with initial-boundary value conditions

$$
\left\{\begin{array}{l}
i q_{t}+\alpha\left(q_{x x}+2|q|^{2} q\right)+i \beta\left(q_{x x x}+6|q|^{2} q_{x}\right)=0, \quad x \in(-L, L), \quad t \in\left(t_{0}, T\right), \\
q\left(x, t_{0}\right)=q_{0}(x), \quad x \in[-L, L], \\
q(-L, t)=q(L, t), \quad t \in\left[t_{0}, T\right],
\end{array}\right.
$$

where $q=q(x, t)$ is a complex envelope field, $\alpha$ and $\beta$ are real constants for the second- and third-order dispersion coefficients, respectively. For $\beta=0$, the Hirota equation (2) becomes a nonlinear Schrödinger (NLS) equation, whereas $\alpha=0$, the Hirota equation (2) reduces to the complex modified KdV equation [22]

$$
q_{t}+\beta\left(q_{x x x}+6|q|^{2} q_{x}\right)=0 .
$$

The rest of this paper is arranged as follows. In Sec. 2, we simply introduce the PINN scheme, and apply it to investigate the data-driven soliton, breather, and rogue wave solutions of Eq. (2) with $\alpha=1, \beta=0.01$. In Sec. 3, we introduce the PINNs scheme, and apply it to study the data-driven parameter discovery of Eq. (2) with the aid of solitons. Finally, we give some conclusions and discussions.

\section{The PINN scheme for the data-driven solutions}

\subsection{The PINNs scheme}

In this subsection, we would like to simply introduce the PINN deep learning method [46] for the data-driven solutions. The main idea of the PINN deep learning method is to use a deep neural network to fit the solutions of Eq. (2). Let $q(x, t)=u(x, t)+i v(x, t)$ with $u(x, t), v(x, t)$ being its real and imaginary parts, respectively. The complex-valued PINN $F(x, t)=F_{u}(x, t)+i F_{v}(x, t)$ with $F_{u}(x, t), F_{v}(x, t)$ being its real and imaginary parts, respectively are written as

$$
\begin{aligned}
& F(x, t):=i q_{t}+\alpha\left(q_{x x}+2|q|^{2} q\right)+i \beta\left(q_{x x x}+6|q|^{2} q_{x}\right), \\
& F_{u}(x, t):=-v_{t}+\alpha\left[u_{x x}+2\left(u^{2}+v^{2}\right) u\right]-\beta\left[v_{x x x}+6\left(u^{2}+v^{2}\right) v_{x}\right], \\
& F_{v}(x, t):=u_{t}+\alpha\left[v_{x x}+2\left(u^{2}+v^{2}\right) v\right]+\beta\left[u_{x x x}+6\left(u^{2}+v^{2}\right) u_{x}\right],
\end{aligned}
$$

and proceeded by approximating $q(x, t)$ by a complex-valued deep neural network. In the PINN scheme, the complex-valued neural network $q(x, t)=(u(x, t), v(x, t))$ can be written as

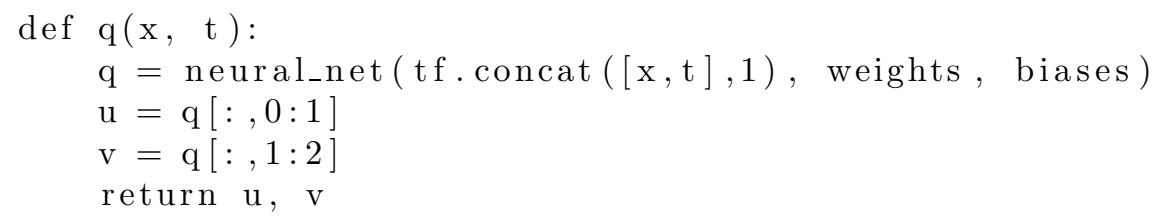

Based on the defined $q(x, t)$, the physics-informed neural network $F(x, t)$ can be taken as

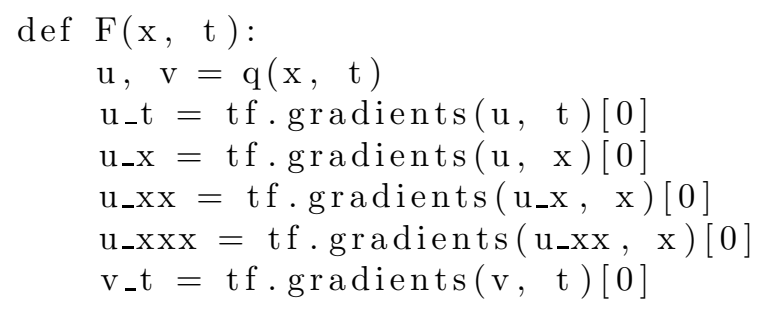




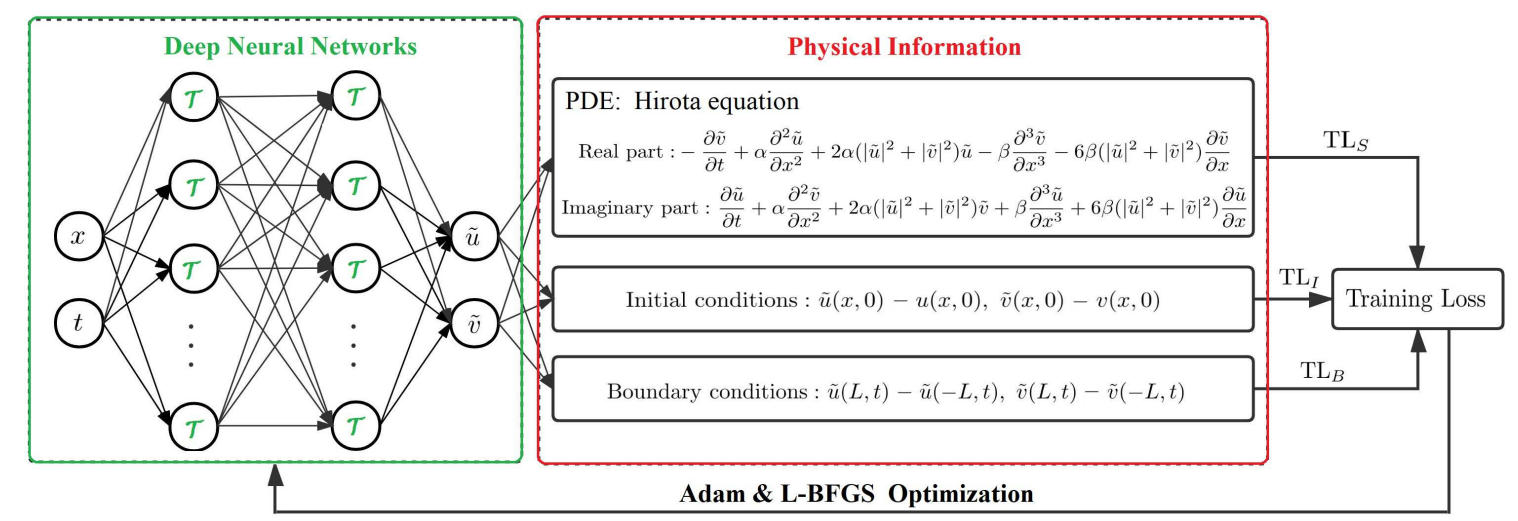

Figure 1: The PINN scheme solving the Hirota equation (2) with the initial and boundary conditions, where the activation function $\mathcal{T}=\tanh (\cdot)$.

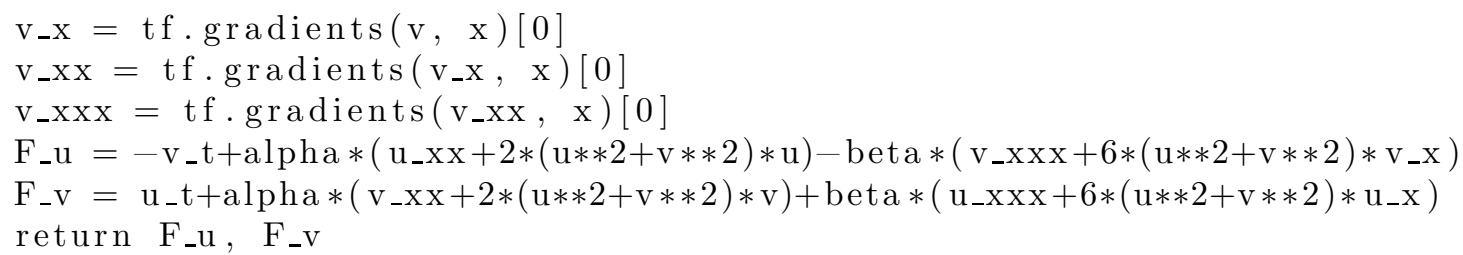

The shared parameters, weights and biases, between the neural network $\tilde{q}(x, t)=u(x, t)+i v(x, t)$ and $F(x, t)=$ $F_{u}(x, t)+i F_{v}(x, t)$ can be learned by minimizing the whole training loss (TL), that is, the sum of the $\mathbb{L}^{2}$-norm training losses of the initial data $\left(\mathrm{TL}_{I}\right)$, boundary data $\left(\mathrm{TL}_{B}\right)$, and the whole equation $F(x, t)\left(\mathrm{TL}_{S}\right)$

$$
\mathrm{TL}=\mathrm{TL}_{I}+\mathrm{TL}_{B}+\mathrm{TL}_{S}
$$

where the mean squared (i.e., $\mathbb{L}^{2}$-norm) errors are chosen for them in the forms

$$
\begin{aligned}
& \mathrm{TL}_{I}=\frac{1}{N_{I}} \sum_{j=1}^{N_{I}}\left(\left|u\left(x_{I}^{j}, t_{0}\right)-u_{0}^{j}\right|^{2}+\left|v\left(x_{I}^{j}, t_{0}\right)-v_{0}^{j}\right|^{2}\right), \\
& \mathrm{TL}_{B}=\frac{1}{N_{B}} \sum_{j=1}^{N_{B}}\left(\left|u\left(-L, t_{B}^{j}\right)-u\left(L, t_{B}^{j}\right)\right|^{2}+\left|v\left(-L, t_{B}^{j}\right)-v\left(L, t_{B}^{j}\right)\right|^{2}\right), \\
& \mathrm{TL}_{S}=\frac{1}{N_{S}} \sum_{j=1}^{N_{S}}\left(\left|F_{u}\left(x_{S}^{j}, t_{S}^{j}\right)\right|^{2}+\left|F_{v}\left(x_{S}^{j}, t_{S}^{j}\right)\right|^{2}\right)
\end{aligned}
$$

with $\left\{x_{I}^{j}, u_{0}^{j}, v_{0}^{j}\right\}_{j=1}^{N_{I}}$ denoting the initial data $\left(q_{0}(x)=u_{0}(x)+i v_{0}(x)\right),\left\{t_{B}^{j}, u\left( \pm L, t_{B}^{j}\right), v\left( \pm L, t_{B}^{j}\right\}_{j=1}^{N_{B}}\right.$ standing for the periodic boundary data, $\left\{x_{S}^{j}, t_{S}^{j}, F_{u}\left(x_{S}^{j}, t_{S}^{j}\right), F_{v}\left(x_{S}^{j}, t_{S}^{j}\right)\right\}_{j=1}^{N_{S}}$ representing the collocation points of $F(x, t)=F_{u}+i F_{v}$ within a spatio-temporal region $(x, t) \in(-L, L) \times\left(t_{0}, T\right]$. All of these sampling points are generated using a space filling Latin Hypercube Sampling strategy [53].

We would like to discuss some data-driven solutions of Eq. (2) by the deep learning method. Here we choose 
a 5-layer deep neural network with 40 neurons per layer and a hyperbolic tangent activation function tanh(·)

$$
\begin{aligned}
A^{j+1} & =\tanh \left(W^{j+1} A^{j}+B^{j+1}\right) \\
& =\left(\tanh \left(\sum_{s=1}^{m_{j}} w_{1 s}^{j+1} a_{s}^{j}+b_{1}^{j+1}\right), \cdots, \tanh \left(\sum_{s=1}^{m_{j}} w_{m_{j+1} s}^{j+1} a_{s}^{j}+b_{m_{j}}^{j+1}\right)\right)^{T}, \quad j=0,1,2, \ldots, M
\end{aligned}
$$

to approximate the learning solutions, where $A^{j}=\left(a_{1}^{j}, a_{2}^{j}, \ldots, a_{m_{j}}^{j}\right)^{T}$ and $B^{j}=\left(b_{1}^{j}, b_{2}^{j}, \ldots, b_{m_{j}}^{j}\right)^{T}$ denote the output and bias column vectors of the $j$-th layer, respectively, $W^{j+1}=\left(w_{k s}^{j+1}\right)_{m_{j+1} \times m_{j}}$ stands for the weight matrix of the $j$-th layer, $A^{0}=(x, t)^{T}, A^{M+1}=(u, v)^{T}$. The real and imaginary parts, $u(x, t)$ and $v(x, t)$, of approximated solution $\tilde{q}(x, t)=u(x, t)+i v(x, t)$ are represented by the two outputs of one neural network (see Fig. 1 for the PINN scheme).

In the following, we consider some fundamental solutions (e.g. soliton, breather, and rogue wave solutions) of Eq. (2) by using the PINNs deep leaning scheme. For the case $\alpha \beta \neq 0$ in Eq. (2), without loss of generality, we can take $\alpha=1, \beta=0.01$.

\subsection{The data-driven bright soliton}

The first example we would like to consider is the fundamental bright soliton of Eq. $(2)[19,22]$

$$
q_{b s}(x, t)=\operatorname{sech}(x-\beta t) e^{i t},
$$

where the third-order dispersion coefficient $\beta$ stands for the wave velocity, and the sign of $\beta$ represents the direction of wave propagation [right-going (left-going) travelling wave soliton for $\beta>0(\beta<0)$ ].

We here choose $L=10, t_{0}=0, T=5$, and will consider this problem by choosing two distinct kinds of initial sample points: In the first case, we will choose the $N_{I}=100$ random sample points from the initial data $q_{b s}(x, t=0)$ with $x \in[-10,10]$. But in the second case, we only choose $N_{I}=5$ sample points from the initial data $q_{b s}(x, t=0)$ with 5 equidistant and symmetric points $x \in\{-5,-2.5,0,2.5,5\}$. In the both cases, we use the same $N_{B}=200$ periodic boundary random sample points and $N_{S}=10,000$ random sample points in the solution region $\left\{\left(x, t, q_{b s}(x, t)\right) \mid(x, t) \in[-10,10] \times[0,5]\right\}$. It is worth mentioning that the $N_{S}=10,000$ sample points are obtained via the Latin Hypercube Sampling strategy [53].

We emulate the first case of initial data by using 10,000 steps Adam and 10,000 steps L-BFGS optimizations such that Figs. 2(a1-a3) and (b1-b3) illustrate the learning results starting from the unperturbated and perturbated ( $2 \%$ noise) training data, respectively. The relative $\mathbb{L}^{2}$-norm errors of $q(x, t), u(x, t)$ and $v(x, t)$, respectively, are $9.3183 \cdot 10^{-3}, 5.3270 \cdot 10^{-2}, 3.8502 \cdot 10^{-2}$ in Figs. $2\left(\right.$ a1-a2), and $7.0707 \cdot 10^{-3}, 2.4057 \cdot 10^{-2}$, $1.6464 \cdot 10^{-2}$ in Figs. 2(b1-a2). Similarly, we use the 20,000 steps Adam and 50,000 steps L-BFGS optimizations for the second case of initial data such that Figs. 2(c1-c3) and (d1-b3) illustrate the learning results starting from the unperturbated and perturbated training data, respectively. The relative $\mathbb{L}^{2}-$ norm errors of $q(x, t)$, $u(x, t)$ and $v(x, t)$, respectively, are $1.8822 \cdot 10^{-2}, 4.9227 \cdot 10^{-2}, 4.0917 \cdot 10^{-2}$ in Figs. $2(\mathrm{c} 1-\mathrm{c} 2)$, and $2.5427 \cdot 10^{-2}$, $3.4825 \cdot 10^{-2}, 2.5983 \cdot 10^{-2}$ in Figs. 2(d1-d2). Notice that those total learning times are (a) $717 \mathrm{~s},(\mathrm{~b}) 741 \mathrm{~s}$, (c) $1255 \mathrm{~s}$, and (d) $1334 \mathrm{~s}$, respectively, by using a Lenovo notebook with a $2.6 \mathrm{GHz}$ six-cores i7 processor and a RTX2060 graphics processor.

Remark. In each step of the L-BFGS optimization, the program is stop at

$$
\frac{|\operatorname{loss}(n)-\operatorname{loss}(n-1)|}{\max (|\operatorname{loss}(n)|,|\operatorname{loss}(n-1)|, 1)}<1.0 \times \text { np.finfo(float).eps, }
$$

where the $\operatorname{loss}(n)$ represents the value of loss function in the $n$-th step L-BFGS optimization, and $1.0 \times$ np.finfo(float).eps represent Machine Epsilon. When the relative error between $\operatorname{loss}(n)$ and $\operatorname{loss}(n-1)$ less than Machine Epsilon, procedure would be stop. This is why the computation times are different for each test by using the same step optimization. 

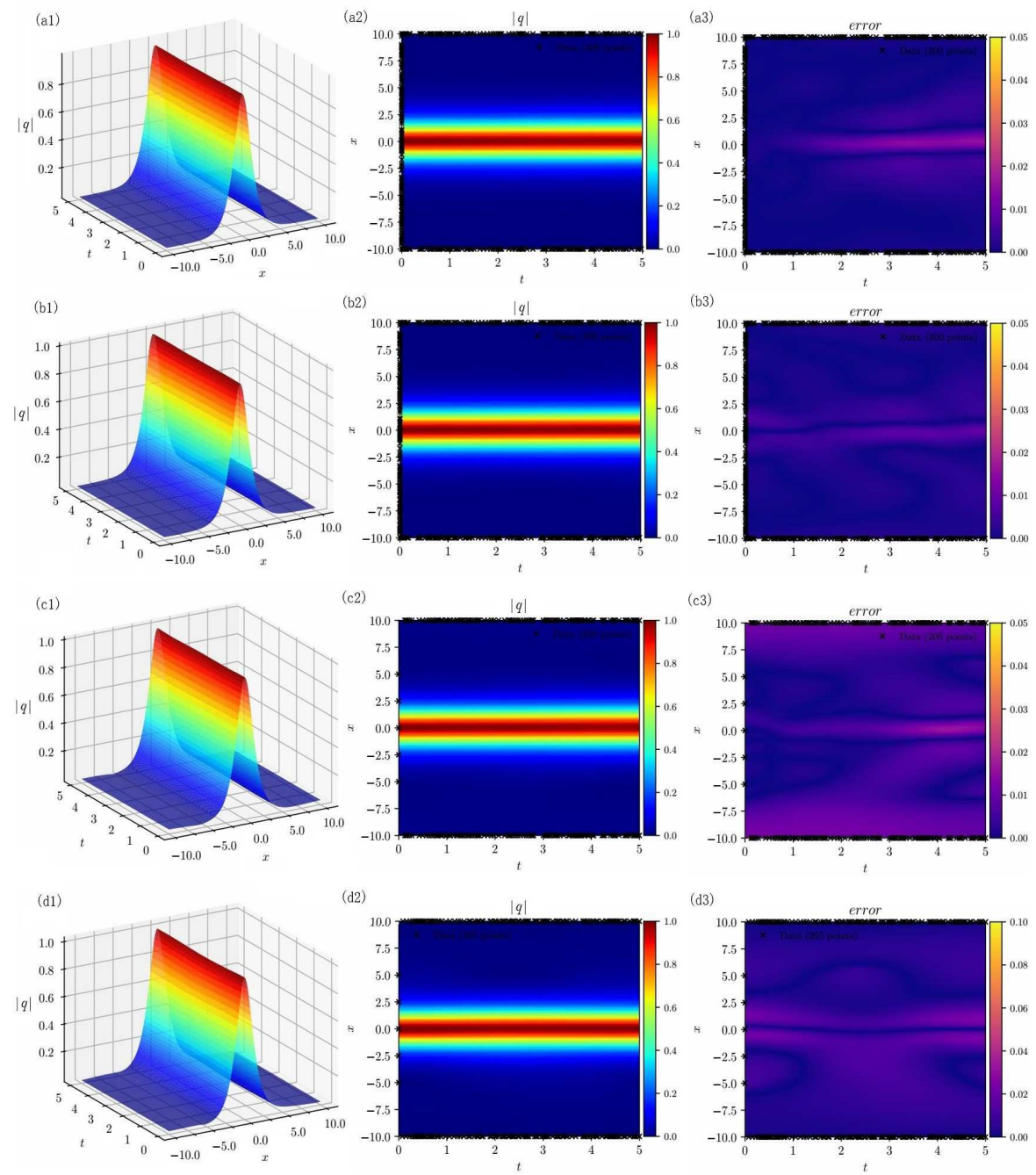

Figure 2: Data-driven soliton of the Hirota equation (2): (a1,a2) and (b1,b2) the learning solutions arising from the unpeturbated and perturbated $(2 \%)$ training data related to the first case of initial data, respectively; (c1,c2) and $(\mathrm{d} 1, \mathrm{~d} 2)$ the learning solutions arising from the unpeturbated and perturbated $(2 \%)$ training data related to the first case of initial data, respectively; (a3, b3, c3, d3) the absolute values of the errors between the modules of exact and learning solutions. The relative $\mathbb{L}^{2}$-norm errors of $q(x, t), u(x, t)$ and $v(x, t)$, respectively, are (a1-a3) $9.3183 \cdot 10^{-3}, 5.3270 \cdot 10^{-2}$, $3.8502 \cdot 10^{-2}$, (b1-b3) $7.0707 \cdot 10^{-3}, 2.4057 \cdot 10^{-2}, 1.6464 \cdot 10^{-2},(\mathrm{c} 1-\mathrm{c} 3) 1.8822 \cdot 10^{-2}, 4.9227 \cdot 10^{-2}, 4.0917 \cdot 10^{-2},(\mathrm{~d} 1-\mathrm{d} 3$ ) $2.5427 \cdot 10^{-2}, 3.4825 \cdot 10^{-2}, 2.5983 \cdot 10^{-2}$.

\subsection{The data-deriven AKM breather solution}

The second example we would like to study is the AKM breather (spatio-temporal periodic pattern) of Eq. (2) [28]

$$
q_{a k m}(x, t)=\frac{\cosh (\omega t-2 i c)-\cos (c) \cos (p \xi)}{\cosh (\omega t)-\cos (c) \cos (p \xi)} e^{2 i t},
$$

where $\xi=x-2 \beta[2+\cos (2 c) t], \omega=2 \sin (2 c), p=2 \sin (c)$, and $c$ is a real constant. The wave velocity and wavenumber of this periodic wave are $2 \beta(2+\cos (2 c))$ and $p$, respectively. This AKM breather differs from the Akhmediev breather (spatial periodic pattern) of the NLS equation because Eq. (2) contains the third-order 

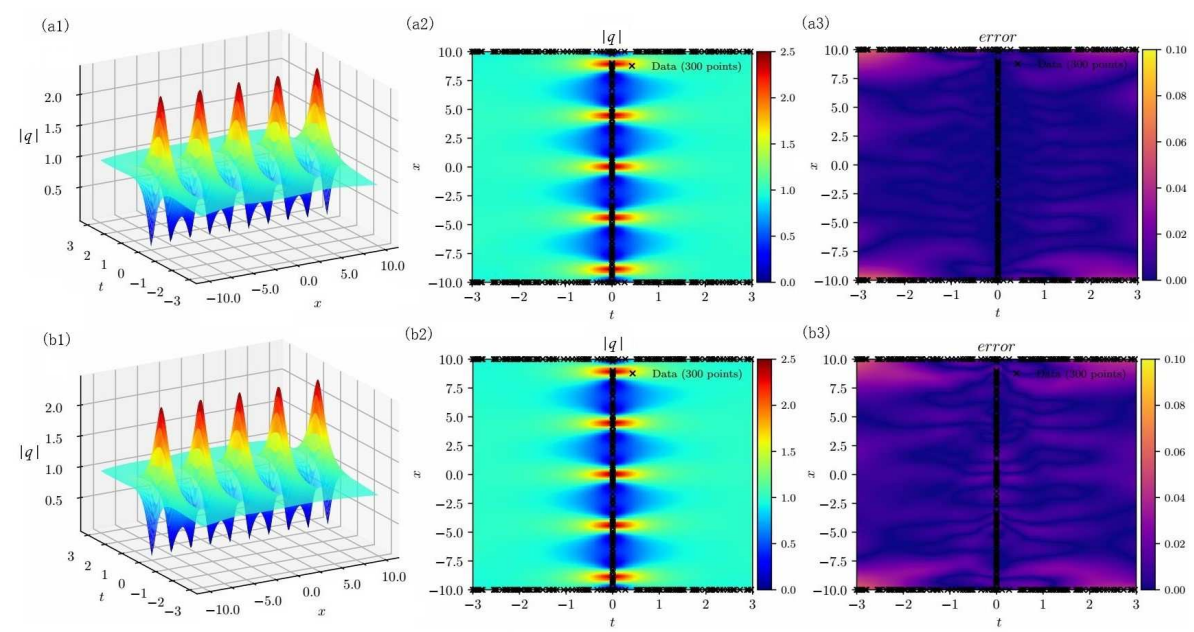

Figure 3: Learning breathers related to the AKM breather (10) of the Hirota equation (2). (a1-a3) the unperturbbated case, (b1-b3) the $2 \%$ perturbated case. The relative $\mathbb{L}^{2}$-norm errors of $q(x, t), u(x, t)$ and $v(x, t)$, respectively, are (a1-a3) $1.1011 \cdot 10^{-2}, 3.5650 \cdot 10^{-2}, 5.0245 \cdot 10^{-2},(\mathrm{~b} 1-\mathrm{b} 3) 1.3458 \cdot 10^{-2}, 5.1326 \cdot 10^{-2}, 7.0242 \cdot 10^{-2}$.

coefficient $\beta$. In this example, we assume $\beta=0.01$ again. When $t \rightarrow \infty,\left|q_{a k m}(x, t)\right|^{2} \rightarrow 1$. If $\beta \rightarrow 0$, we have $\xi \rightarrow x$, and then AKM breather almost becomes the Akhmediev breather.

We here choose $L=10$ and $t \in[-3,3]$, and choose the $N_{I}=100$ random sample points from the initial data $q_{a k m}(x, t=0), N_{B}=200$ random sample points from the periodic boundary data, and $N_{S}=10,000$ random sample points in the solution region $(x, t) \in[-10,10] \times[-3,3]$. We use the 20,000 Adam and 50,000 L-BFGS optimizations to learn the solutions from the unperturbated and perturbated (a $2 \%$ noise) initial data. As a result, Figs. 3 (a1-a3) and (b1-b3) exhibit the leaning results for the unperturbated and perturbated (a $2 \%$ noise) cases, respectively. The relative $\mathbb{L}^{2}$-norm errors of $q(x, t), u(x, t)$ and $v(x, t)$, respectively, are (a) $1.1011 \cdot 10^{-2}, 3.5650 \cdot 10^{-2}, 5.0245 \cdot 10^{-2}$, (b) $1.3458 \cdot 10^{-2}, 5.1326 \cdot 10^{-2}, 7.0242 \cdot 10^{-2}$. The learning times are 2268 s and 1848 s, respectively.

\subsection{The data-driven rogue wave solution}

The third example is a fundamental rogue wave solution of Eq. (2), which can be generated when one takes $c \rightarrow 0$ in the AKM breather (10) in the form [29]

$$
q_{r w}(x, t)=\left[1-\frac{4(1+4 i t)}{4(x-6 \beta t)^{2}+16 t^{2}+1}\right] e^{2 i t} .
$$

As $|x|,|t| \rightarrow \infty,\left|q_{r w}\right| \rightarrow 1$, and $\max _{x, t}|q|=3$.

We here choose $L=2.5$ and $t \in[-0.5,0.5]$, and consider $q_{r w}(x, t=-0.5)$ as the initial condition. We still choose $N_{I}=100$ random sample points from the initial data $q_{r w}(x, t=-0.5), N_{B}=200$ random sample points from the periodic boundary data, and $N_{S}=10,000$ random sample points in the solution region $(x, t) \in$ $[-2.5,2.5] \times[-0.5,0.5]$. We use the 20,000 steps Adam and 50,000 steps L-BFGS optimizations to learn the rogue wave solutions from the unperturbated and perturbated (a $2 \%$ noise) initial data, respectively. As a result, Figs. 4(a1-a3) and (b1-b3) exhibit the leaning results for the unperturbated and perturbated (a $2 \%$ noise) cases, respectively. The relative $\mathbb{L}^{2}$-norm errors of $q(x, t), u(x, t)$ and $v(x, t)$, respectively, are (a) $6.7597 \cdot 10^{-3}$, $8.8414 \cdot 10^{-3}, 1.6590 \cdot 10^{-2}$, (b) $3.9537 \cdot 10^{-3}, 5.8719 \cdot 10^{-3}, 9.0493 \cdot 10^{-3}$. The learning times are $1524 \mathrm{~s}$ and $1414 \mathrm{~s}$, respectively. 

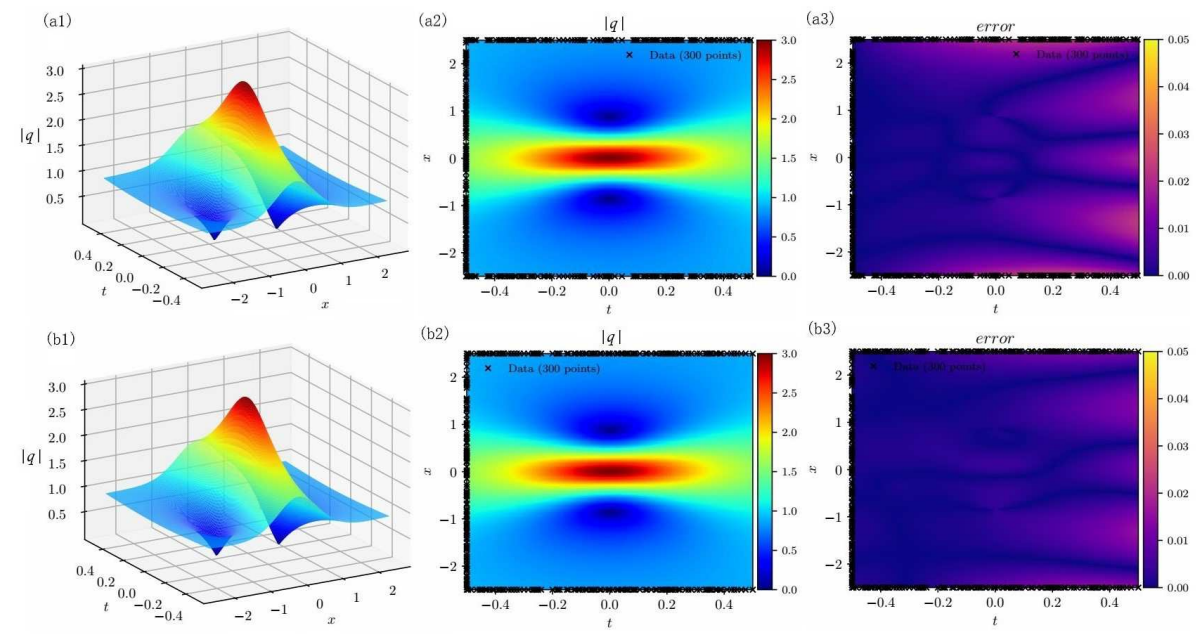

Figure 4: Learning rogue wave solution related to Eq. (11) of the Hirota equation (2). (a1-a3) the unperturbated case, (b1-b3) the $2 \%$ perturbated case. The relative $\mathbb{L}^{2}$-norm errors of $q(x, t), u(x, t)$ and $v(x, t)$, respectively, are (a1-a3) $6.7597 \cdot 10^{-3}, 8.8414 \cdot 10^{-3}, 1.6590 \cdot 10^{-2},(\mathrm{~b} 1-\mathrm{b} 3) 3.9537 \cdot 10^{-3}, 5.8719 \cdot 10^{-3}, 9.0493 \cdot 10^{-3}$.

\section{The PINNs scheme for the data-driven parameter discovery}

In this section, we apply the PINNs deep learning method to study the data-driven parameter discovery of the Hirota equation (2). In the following, we use the deep learning method to identify the parameters $\alpha$ and $\beta$ in the Hirota equation (2). Moreover, we also use this method to identify the parameters of the high-order terms of Eq. (2).

\subsection{The data-driven parameter discovery for $\alpha$ and $\beta$}

Here we would like to use the PINNs deep learning method to identify the coefficients $\alpha, \beta$ of second- and third-order dispersive terms in the Hirota equation

$$
i q_{t}+\alpha\left(q_{x x}+2|q|^{2} q\right)+i \beta\left(q_{x x x}+6|q|^{2} q_{x}\right)=0,
$$

where $\alpha, \beta$ are the unknown real-valued parameters.

Let $q(x, t)=u(x, t)+i v(x, t)$ with $u(x, t), v(x, t)$ being its real and imaginary parts, respectively, and the PINNs $F(x, t)=F_{u}(x, t)+i F_{v}(x, t)$ with $F_{u}(x, t), F_{v}(x, t)$ being its real and imaginary parts, respectively, be

$$
\begin{aligned}
& F(x, t):=i q_{t}+\alpha\left(q_{x x}+2|q|^{2} q\right)+i \beta\left(q_{x x x}+6|q|^{2} q_{x}\right), \\
& F_{u}(x, t):=-v_{t}+\alpha\left[u_{x x}+2\left(u^{2}+v^{2}\right) u\right]-\beta\left[v_{x x x}+6\left(u^{2}+v^{2}\right) v_{x}\right], \\
& F_{v}(x, t):=u_{t}+\alpha\left[v_{x x}+2\left(u^{2}+v^{2}\right) v\right]+\beta\left[u_{x x x}+6\left(u^{2}+v^{2}\right) u_{x}\right],
\end{aligned}
$$

Then the deep neural network is used to learn $\{u(x, t), v(x, t)\}$ and parameters $(\alpha, \beta)$ by minimizing the mean squared error loss

$$
\mathrm{TL}=\mathrm{TL}_{q}+\mathrm{TL}_{p}
$$



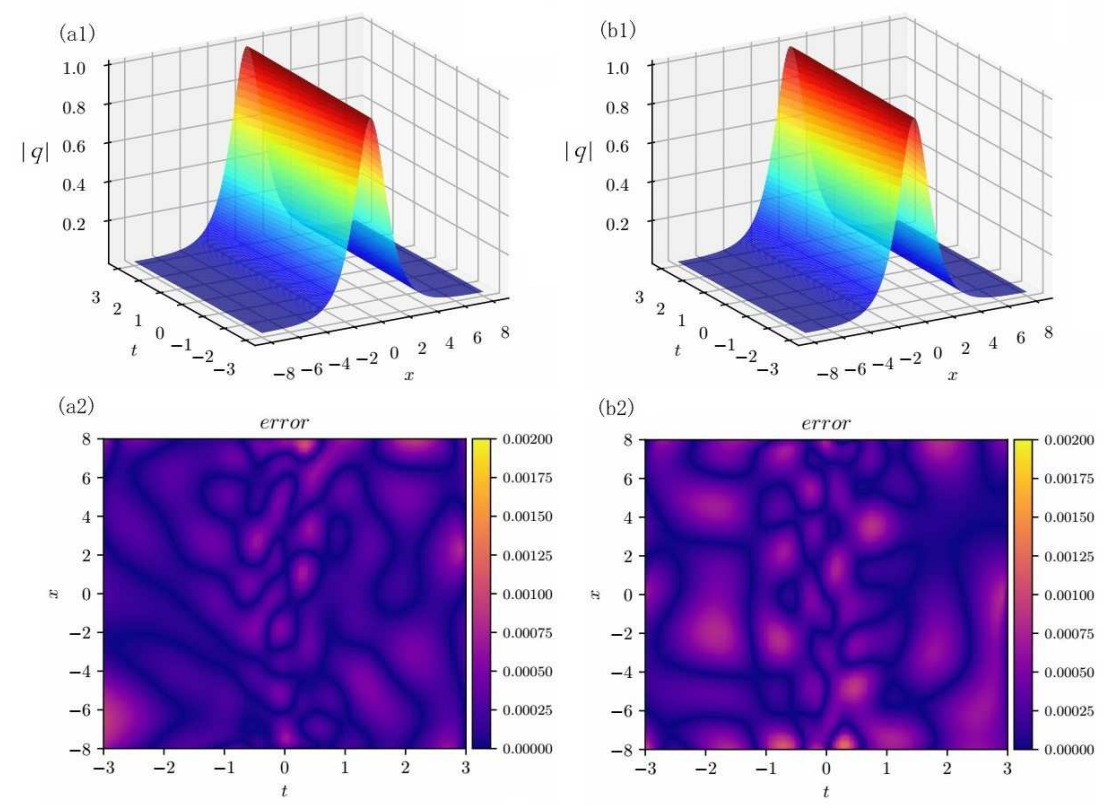

Figure 5: Data-driven parameter discovery of $\alpha$ and $\beta$ in the sense of soliton (8). (a1-a2) soliton without perturbation. (b1-b2) soliton with a $2 \%$ noise. (a2, b2) the absolute value of difference between the modules of exact and learning solitons. The relative $\mathbb{L}^{2}$-norm errors of $q(x, t), u(x, t)$ and $v(x, t)$, respectively, are (a1-a2) $7.0371 \cdot 10^{-4}, 1.0894 \cdot 10^{-3}$, $1.0335 \cdot 10^{-3},(\mathrm{~b} 1-\mathrm{b} 2) 9.4420 \cdot 10^{-4}, 1.4055 \cdot 10^{-3}, 1.2136 \cdot 10^{-3}$.

with

$$
\begin{aligned}
& \mathrm{TL}_{q}=\frac{1}{N_{p}} \sum_{j=1}^{N_{p}}\left(\left|u\left(x^{j}, t^{j}\right)-u^{j}\right|^{2}+\left|v\left(x^{j}, t^{j}\right)-v^{j}\right|^{2}\right), \\
& \mathrm{TL}_{p}=\frac{1}{N_{p}} \sum_{j=1}^{N_{p}}\left(\left|F_{u}\left(x^{j}, t^{j}\right)\right|^{2}+\left|F_{v}\left(x^{j}, t^{j}\right)\right|^{2}\right),
\end{aligned}
$$

where $\left\{x^{j}, t^{j}, u^{j}, v^{j}\right\}_{i=1}^{N_{p}}$ represents the training data on the real part and imaginary part of exact solution $u(x, t), v(x, t)$ given by Eq. (8) with $\alpha=1, \beta=0.5$ in $(x, t) \in[-8,8] \times[-3,3]$, and $u\left(x^{j}, t^{j}\right), v\left(x^{j}, t^{j}\right)$ are real and imaginary parts of the approximate solution $q(x, t)=u(x, t)+i v(x, t)$.

To study the data-driven parameter discovery of the Hirota equation (2) for $\alpha, \beta$, we generate a training dataset by using the Latin Hypercube Sampling strategy to randomly select randomly choosing $N_{q}=10,000$ points in the solution region arising from the exact bright soliton (8) with $\alpha=1, \beta=0.5$ and $(x, t) \in[-8,8] \times[-3,3]$. Then the obtained data-set is applied to train an 8-layer deep neural network with 20 neurons per layer and a same hyperbolic tangent activation function to approximate the parameters $\alpha, \beta$ in terms of minimizing the mean squared error loss given by Eqs. (14) and (15) starting from $\alpha=\beta=0$ in Eq. (17). We here use the 20,000 steps Adam and 50,000 steps L-BFGS optimizations.

Table 1 illustrates the learning parameters $\alpha, \beta$ in Eq. (12) under the cases of the data without perturbation and a $2 \%$ perturbation, and their errors of $\alpha, \beta$ are $3.85 \times 10^{-5}, 7.48 \times 10^{-5}$ and $3.31 \times 10^{-4}, 2.89 \times 10^{-4}$, respectively. Fig. 5 exhibits the learning solutions and the relative $\mathbb{L}^{2}$-norm errors of $q(x, t), u(x, t)$ and $v(x, t)$ : (a1-a2) $7.0371 \cdot 10^{-4}, 1.0894 \cdot 10^{-3}, 1.0335 \cdot 10^{-3}$; (b1-b2) $9.4420 \cdot 10^{-4}, 1.4055 \cdot 10^{-3}, 1.2136 \cdot 10^{-3}$, where the training times are (a1-a2) 1510s and (b1-b2) 3572s, respectively. 
Table 1: Comparisons of $\alpha, \beta$ and their errors in the different training data-set via deep learning.

\begin{tabular}{cccccc}
\hline \hline Case & Solution & $\alpha$ & error of $\alpha$ & $\beta$ & error of $\beta$ \\
\hline 1 & exact soliton & 1 & 0 & 0.5 & 0 \\
2 & soliton without perturbation & 1.00004 & $3.85 \times 10^{-5}$ & 0.05008 & $7.48 \times 10^{-5}$ \\
3 & soliton with a 2\% perturbation & 0.99967 & $3.31 \times 10^{-4}$ & 0.05029 & $2.89 \times 10^{-4}$ \\
\hline \hline
\end{tabular}
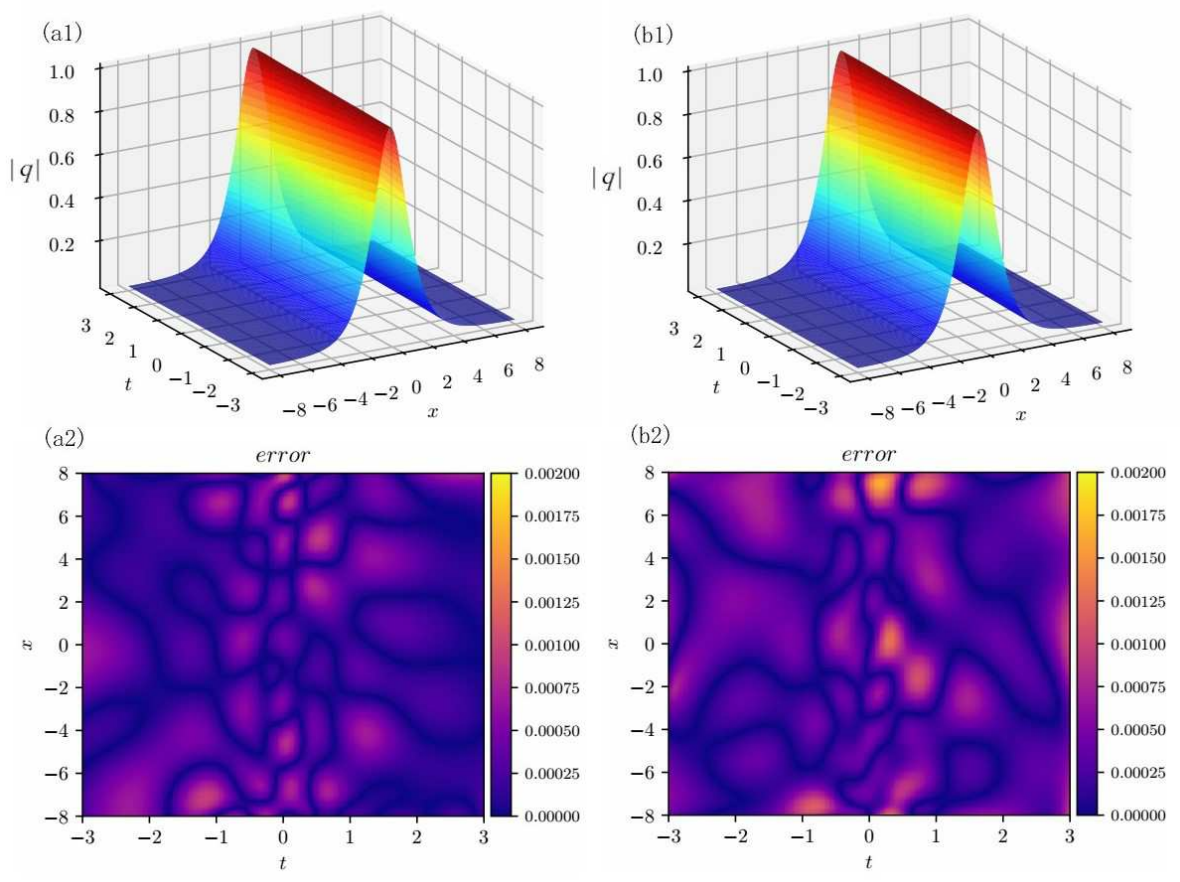

Figure 6: Data-driven parameter discovery of $\mu$ and $\nu$ in the sense of soliton (8). (a)(b) display the learning result under soliton data set. (a1-a2) are calculated without perturbation. (b1-b2) are calculated with 2\% perturbation. (a2) and (b2) exhibit absolute value of difference between real solution and the function represented by the neural network. The relative $\mathbb{L}^{2}$-norm error of $q(x, t), u(x, t)$ and $v(x, t)$, respectively, are (a1-a2) $8.0153 \cdot 10^{-4}, 1.0792 \cdot 10^{-3}, 1.2177 \cdot 10^{-3}$, (b1-b2) $1.0770 \cdot 10^{-3}, 1.6541 \cdot 10^{-3}, 1.3370 \cdot 10^{-3}$.

\subsection{The data-driven parameter discovery for $\mu$ and $\nu$}

In what follows, we will study the learning coefficients of the high-order term in Eq. (2) via the deep learning method. We consider the Hirota equation (2) with two parameters in the form

$$
i q_{t}+q_{x x}+2|q|^{2} q+\frac{i}{2}\left(\mu q_{x x x}+\nu|q|^{2} q_{x}\right)=0,
$$

where $\mu$ and $\nu$ are the unknown real constants of higher-order dispersion and nonlinear terms, respectively.

Let $q(x, t)=u(x, t)+i v(x, t)$ with $u(x, t), v(x, t)$ being its real and imaginary parts, respectively, and the 
Table 2: Comparisons of $\mu, \nu$ and their errors in the different training data-set via deep learning.

\begin{tabular}{cccccc}
\hline \hline Case & Solution & $\mu$ & error of $\mu$ & $\nu$ & error of $\nu$ \\
\hline 1 & exact soliton & 1 & 0 & 1 & 0 \\
2 & soliton without perturbation & 1.00370 & $3.69 \times 10^{-3}$ & 6.03143 & $3.14 \times 10^{-2}$ \\
3 & soliton with a 2\% perturbation & 0.98159 & $1.84 \times 10^{-2}$ & 5.88733 & $1.13 \times 10^{-1}$ \\
\hline \hline
\end{tabular}

PINNs $F(x, t)=F_{u}(x, t)+i F_{v}(x, t)$ with $F_{u}(x, t), F_{v}(x, t)$ being its real and imaginary parts, respectively, be

$$
\begin{aligned}
& F(x, t):=i q_{t}+q_{x x}+2|q|^{2} q+\frac{i}{2}\left(\mu q_{x x x}+\nu|q|^{2} q_{x}\right), \\
& F_{u}(x, t):=-v_{t}+u_{x x}+2\left(u^{2}+v^{2}\right) u-\frac{1}{2}\left[\mu v_{x x x}+\nu\left(u^{2}+v^{2}\right) v_{x}\right], \\
& F_{v}(x, t):=u_{t}+v_{x x}+2\left(u^{2}+v^{2}\right) v+\frac{1}{2}\left[\mu u_{x x x}+\nu\left(u^{2}+v^{2}\right) u_{x}\right] .
\end{aligned}
$$

Then the deep neural network is used to learn $\{u(x, t), v(x, t)\}$ and parameters $(\mu, \nu)$ by minimizing the mean squared error loss given by Eqs. (14) and (15).

To illustrate the learning ability, we still use an 8-layer deep neural network with 20 neurons per layer. We choose $N_{q}=10,000$ sample points by the same way in the interior of solution region. The 20,000 steps Adam and 50,000 steps L-BFGS optimizations are used in the training process. Table 2 exhibits the training value and value errors of $\mu$ and $\nu$ in different training data set. And the results of neural network fitting exact solution are shown in Fig. 6. The training times are (a1-a2) 1971s and (b1-b2) 1990s, respectively.

\section{Conclusions and discussions}

In conclusion, we have explored the data-driven solutions and parameter discovery of the third-order nonlinear Schrödinger equation (alias the Hirota equation) via the deep learning method. We use the physics-informed neural networks (PINNs) deep learning method to study the data-driven fundamental solutions (e.g., soliton, breather, and rogue waves) of the Hirota equation, where the two types of the unperturbated and perturbated (a $2 \%$ noise) training data are considered. Moreover, we use the PINNs deep learning to study the data-driven

discovery of parameters appearing in the Hirota equation under the sense of its solitons. The PINN scheme can also be used to study the rogue waves of other nonlinear wave equations.

\section{Acknowledgments}

This work is supported by the NSFC under Grant Nos. 11925108 and 11731014.

\section{Data availability statement}

The data that support the findings of this study are available upon reasonable request from the authors.

\section{References}

[1] G. A. Askar'yan, Effects of the gradient of a strong electromagnetic beam on electrons and atoms, Zh. Eksperim. i Teor. Fiz. 42 (1962) 1567 [Sov. Phys. JETP 15 (1962) 1088].

[2] M. Hercher, Laser-induced change in transparent media, J Opt. Soc. Am. 54 (1964) 563.

[3] R. Chaio, E. M. Garmire, C.H. Townes, Self-trapping of optical beams, Phys. Rev. Lett. 13 (1964) $479-482$.

[4] V. I. Talanov, Self-focusing of electromagnetic waves in nonlinear media, Radiophysics 8 (1964) $254-257$. 
[5] E. P. Gross, Structure of quantized vortex, Nuovo Cim. 20 (1961) 454-461.

[6] L. P. Pitaevskii, Vortex lines in an imperfect Bose gas, Sov. Phys. JETP 13 (1961) 451-454.

[7] P. L. Kelley, Self-focusing of optical beams, Phys. Rev. Lett. 15 (1965) 1005-1008.

[8] V. E. Zakharov, Stability of periodic waves of finite amplitude on the surface of a deep fluid, J. Appl. Mech. Tech. Phys. 9 (1968) 86-94.

[9] J. H. Marburger, Self-focusing: Theory, Prog. Quant. Electr. 4 (1975) 35-110.

[10] S. G. Thornhill and D. ter Haar, Langmuir turbulence and modulational instability, Phys. Rep. 43 (1978) $43-99$.

[11] C. Sulem, P.-L. Sulem, The Nonlinear Schrödinger Equation: Self-focusing and Wave Collapse (Springer, New York, 1999).

[12] M. J. Ablowitz, B. Prinari, A. D. Trubatch, Discrete and Continuous Nonlinear Schrödinger Systems (Cambridge University Press, Cambridge, 2003).

[13] B. A. Malomed, D. Mihalache, F. Wise, L. Torner, Spatiotemporal optical solitons, J. Opt. B: Quantum Semiclass. Opt. 7 (2005) R53.

[14] A. R. Osborne, Nonlinear Ocean Waves (New York: Academic, 2009).

[15] Z. Yan, Financial rogue waves, Commun. Theor. Phys. 54 (2010) 947-949.

[16] G. P. Agrawal, Nonlinear Fiber Optics (5th edn.) (New York, Academic Press, 2012).

[17] Y. S. Kivshar, G. P. Agrawal, Optical Solitons: from Fibers to Photonic Crystals (New York, Academic Press, 2013).

[18] L. Pitaevskii, S. Stringari, Bose-Einstein Condensation and Superfluidity (Oxford, Oxford University Press, 2016).

[19] Y. Kodama, Optical solitons in a monomode fiber, J. Stat. Phys. 39, 597 (1985).

[20] Y. Kodama and A. Hasegawa, Nonlinear pulse propagation in a monomode dielectric guide, IEEE J. Quantum Electron. 23, 510 (1987).

[21] Z. Yan and C. Dai, Optical rogue waves in the generalized inhomogeneous higher-order nonlinear Schrödinger equation with modulating coefficients, J. Opt. 15, 064012 (2013).

[22] R. Hirota, Exact envelope-soliton solutions of a nonlinear wave equation, J. Math. Phys. 14, 805 (1973).

[23] R Gogoi, L Kalita and N Devi, Stability analysis and investigation of higher order Schrödinger equation for strongly dispersive ion-acoustic wave in plasma, J. Phys.: Conf. Ser 208 (2010) 012085.

[24] K. Trulsen and K. B. Dysthe, A modified nonlinear Schrödinger equation for broader bandwidth gravity waves on deep water, Wave Motion 24, 281-289 (1996).

[25] W. Craig, P. Guyenne, and C. Sulem, Hamiltonian higher-order nonlinear Schrödinger equations for broader-banded waves on deep water, Eur. J. Mech. B/Fluids 32, 22-31 (2012).

[26] R. K. Dodd, R. K. Bullough,Families of multisoliton solutions obtained by the inverse method, Lett. Nuovo Cimento, 13 (1975) 313-318.

[27] G. Zhang, S. Chen, and Z. Yan, Focusing and defocusing Hirota equations with non-zero boundary conditions: Inverse scattering transforms and soliton solutions, Commun Nonlinear Sci Numer Simulat 80 (2020) 104927.

[28] N. N. Akhmediev, V. I. Korneev, N. V. Mitskevich, Modulation instability of a continuous signal in an optical fiber taking into account third-order dispersion, Radiophys. Quantum. Electron. 33 (1990) 95-100.

[29] A. Ankiewicz, J. M. Soto-Crespo, and N. Akhmediev, Rogue waves and rational solutions of the Hirota equation. Phys. Rev. E 81, 046602 (2010).

[30] Y. Tao and J. He, Multisolitons, breathers, and rogue waves for the Hirota equation generated by the Darboux transformation, Phys. Rev. E 85, 026601 (2012).

[31] Y. Yang, Z. Yan, and B. A. Malomed, Rogue waves, rational solitons, and modulational instability in an integrable fifth-order nonlinear Schrödinger equation, Chaos 25, 103112 (2015).

[32] Y. Yang, Z. Yan, and B. A. Malomed, Rogue waves, rational solitons, and modulational instability in an integrable fifth-order nonlinear Schrödinger equation, Chaos 25, 103112 (2015).

[33] S. Chen and Z. Yan, The Hirota equation: Darboux transform of the Riemann-Hilbert problem and higher-order rogue waves, Appl. Math. Lett. 95, 65 (2019).

[34] L. Wang, Z. Yan, and B. Guo, Numerical analysis of the Hirota equation: Modulational instability, breathers, rogue waves, and interactions, Chaos 30, 013114 (2020).

[35] Y. LeCun, Y. Bengio, and G. Hinton, Deep learning, Nature 521, 436 (2015).

[36] I. Goodfellow, Y. Bengio, and A. Courville, Deep learning (MIT Press, 2016).

[37] M. Dissanayake and N. Phan-Thien, Neural-network-based approximations for solving partial differential equations, Commun. Numer. Methods Eng. 10, 195-201 (1994). 
[38] I. E. Lagaris, A. Likas, and D. I. Fotiadis, Artificial neural networks for solving ordinary and partial differential equations, IEEE Trans. Neural Networks 9, 987-1000 (1998).

[39] S. H. Rudy, S.L. Brunton, J. L. Proctor, J. N. Kutz, Data-driven discovery of partial differential equations, Sci. Adv. 3, e1602614 (2017).

[40] M. Raissi, P. Perdikaris, G. E. Karniadakis, Machine learning of linear differential equations using Gaussian processes, J. Comput. Phys. 348, 683-693 (2017).

[41] M. Raissi and G.E. Karniadakis, Hidden physics models: machine learning of nonlinear partial differential equations, J. Comput. Phys. 357 (2018) 125-141.

[42] J. Sirignano and K. Spiliopoulos, DGM: A deep learning algorithm for solving partial differential equations, J. Comput. Phys. 375, 1339-1364 (2018).

[43] J. Han, A. Jentzen, and W. E, Solving high-dimensional partial differential equations using deep learning, PNAS 115 (2018) 8505-8510.

[44] G. Pang, L. Lu, and G. E. Karniadakis, fPINNs: Fractional physics-informed neural networks, SIAM J. Sci. Comput. 41, A2603-A2626 (2019).

[45] D. Zhang, L. Lu, L. Guo, and G. E. Karniadakis, Quantifying total uncertainty in physics- informed neural networks for solving forward and inverse stochastic problems, J. Comput. Phys. 397, 108850 (2019).

[46] M. Raissi, P. Perdikaris, and G. E. Karniadakis. Physics-informed neural networks: A deep learning framework for solving forward and inverse problems involving nonlinear partial differential equations, J. Comput. Phys. 378 (2019) 686.

[47] Z. Long, Y. Lu, and B. Dong, PDE-Net 2.0: learning PDEs from data with a numeric-symbolic hybrid deep network, J. Comput. Phys. 399, 108925 (2019).

[48] M. Raissi, A. Yazdani, and G. E. Karniadakis, Hidden fluid mechanics: Learning velocity and pressure fields from flow visualizations, Science 367 (2020) 1026-1030.

[49] A. A. Chattopadhyay, Y. Guan, and P. Hassanzadeh, Data-driven subgrid-scale modeling of forced Burgers turbulence using deep learning with generalization to higher Reynolds numbers via transfer learning, Phys. Fluids 33 (2021) 031702.

[50] Z. Zhou and Z. Yan, Solving forward and inverse problems of the logarithmic nonlinear Schrödinger equation with PT-symmetric harmonic potential via deep learning, Phys. Lett. A 387 (2021) 127010.

[51] L. Wang and Z. Yan, Data-driven rogue waves and parameter discovery in the defocusing NLS equation with a potential using the PINN deep learning, arXiv: 2012.09984 (2020).

[52] L. Lu, X. Meng, Z. Mao, G. E. Karniadakis, DeepXDE: A deep learning library for solving differential equations, . SIAM Rev. 63 (2021) 208-228

[53] M. Stein, Large sample properties of simulations using Latin hypercube sampling, Technometrics 29 (1987) $143-151$. 